\title{
The Network of Pro-Inflammatory Factors CDI47, DcR3, and IL33 in the Development of Kawasaki Disease
}

\section{Yanqi Qi* \\ Jiawen $\mathrm{Xu}^{*}$ \\ Zhe Lin* \\ Yijing Tao (D) \\ Fenglei Zheng \\ Yujia Wang \\ Yameng Sun \\ Songling Fu \\ Wei Wang \\ Chunhong Xie \\ Yiying Zhang \\ Fangqi Gong}

Department of Cardiology, Children's Hospital, Zhejiang University School of Medicine, National Clinical Research Center for Child Health, Hangzhou, 3 10052, People's Republic of China

*These authors contributed equally to this work
Correspondence: Fangqi Gong Department of Cardiology, Children's Hospital, Zhejiang University School of Medicine, National Clinical Research

Center for Child Health, No. 3333

Binsheng Road, Hangzhou, 310052,

People's Republic of China

$\mathrm{Tel} / \mathrm{Fax}+86-57 \mathrm{I}-88873008$

Email gongfangqi@zju.edu.cn
Introduction: Kawasaki disease (KD) is an acute febrile systemic vasculitis, but the etiology remains unknown. We studied serum levels of CD147, DcR3, and IL33 in different stages of KD to explore the value of CD147, DcR3, and IL33 in the pathophysiology of KD. Methods: We measured serum levels of CD147, DcR3, and IL33 by enzyme-linked immunosorbent assay (ELISA) at different stages with $71 \mathrm{KD}$ patients and 66 healthy control children. We apply for network tools GeneMANIA and Cytoscape APP to analyze the functions of these pro-inflammatory factors at the gene and protein level.

Results: Serum levels of CD147, DcR3, and IL33 were significantly increased in KD patients before IVIG treatment. Serum levels of CD147, DcR3, and IL33 gradually decreased over time after the treatment of IVIG. Eight cases were IVIG non-responders, while nine KD patients got CALs, but they did not overlap. And there were no statistical differences between group IVIG responders and IVIG non-responders or between groups without CALs and with CALs. We explored the functions of CD147, DcR3, and IL33 from GeneMANIA and Cytoscape APP and found these third pro-inflammatory factors were coexpressed, physical interactions, genetic interactions with other KD-related factors.

Conclusion: CD147, DcR3, and IL33 are involved in the pathophysiology of KD, which provides novel evidence for diagnosing and treating KD with their inhibitors.

Keywords: Kawasaki disease, coronary artery lesions, CD147, DcR3, IL33, PI3K/AKT pathway

\section{Introduction}

Kawasaki disease (KD), as an acute, febrile systemic vasculitis, its pathogenesis gets more and more attention on account of its severe complications such as coronary artery aneurysm (CAA), thrombosis, myocardial infarction, even sudden death. The mechanism of KD remains unknown, despite several decades of ongoing studies. $^{1}$ The AHA scientific statement ${ }^{1}$ says that: in the early stage in Kawasaki disease, activation of the immune system starts, which with high numbers of activated, circulating neutrophils and activation of cytokines, including interleukin(IL)1, IL6, tumor necrosis factor (TNF) signaling pathways. ${ }^{2}$ Simultaneously, both pro-inflammatory and regulatory $\mathrm{T}$ cells can be found in the circulation. ${ }^{3}$ However, no detailed profile has told us the crucial role of these factors.

The cluster of differentiation (CD)147, also called Basigin (BSG), belongs to the immunoglobulin superfamily. ${ }^{4} \mathrm{CD} 147$ is a potent inducer of angiogenic factors such as VEGF. ${ }^{5}$ It induced the up-regulation of VEGF-derived foam cells, which 
may further promote intraplaque angiogenesis through the PI3K/AKT pathway. ${ }^{6}$ A study indicated elevated CD147 in pressure-overloaded exacerbated $\mathrm{RV}$ dysfunction while suppressing CD147, and its ligand Cyclophilin $\mathrm{A}(\mathrm{CyPA})$ can meliorate RV dysfunction in PH model animals. ${ }^{7}$ What is more, CD147 plays a role in mediating angiogenesis in psoriatic arthritis. ${ }^{8}$

Decoy receptor3 (DcR3), which belongs to the TNFR superfamily, also named TNFRSF6B, is a soluble receptor lacking transmembrane domains. ${ }^{9}$ The overexpression of DcR3 blocks the suppression of apoptosis in some malignant cells by interfering with the binding of VEGI, FasL, and LIGHT to receptors. ${ }^{10}$ Coronary artery disease (CAD) patients requiring coronary artery bypass grafting have high circulating levels of TL1A and DcR3, which may thus be valuable biomarkers for diagnosing severe CAD. ${ }^{11}$ A study demonstrated that DCR3 inhibited inflammatory factor expression levels by regulating the PI3K/AKT signaling pathway. ${ }^{12}$ Taken together, DCR3 may be a promising drug for treating coronary heart disease via regulating inflammation and apoptosis through the PI3K/ AKT signaling pathway. ${ }^{12}$

Interleukin (IL) 33 is a member of the IL1 family that acts by its receptor ST2, and it was described as an immunomodulatory cytokine powerfully inducing $\mathrm{T}$ helper cells type2 (Th2) cytokine production initially. ${ }^{13,14}$ IL33 produces different pro-inflammatory cytokines by directly stimulating human mast cells and improving these cells' survival. ${ }^{15,16}$ What is more, endothelial cells are also the cellular target for IL33. It upregulates the urokinase-type plasminogen activator (u-PA) in endothelial cells and promotes angiogenesis. ${ }^{17}$ Bone marrow-derived mast cells (BMMCs) produce IL6, IL13, and tumor necrosis factor (TNF)- $\alpha$ upon IL33 stimulation and depend on MK2/3-mediated activation of the PI3K/AKT pathways. ${ }^{18}$

As it is known, vascular endothelial cells (VECs) perform a crucial function in the onset and progression of $\mathrm{KD}$. Changes in the serum vascular endothelial growth factor (VEGF) level suggest that the PTEN/PI3K/VEGF signaling pathway plays a role in developing KD. ${ }^{19}$ KEGG PATHWAY Database described the PI3K/AKT signaling pathway (map04151) in detail. The PI3K-Akt signaling pathway is activated by many cellular stimuli or toxic insults and regulates fundamental cellular functions such as transcription, translation, proliferation, growth, and survival. ${ }^{20}$ Coincidently, CD147, DcR3, and IL33 may regulate VECs pathogenesis and are associated with the PI3K/AKT pathway.
CD147 may be necessary to inhibit autophagy via the PI3K/ Akt/mTOR pathway. ${ }^{21}$ Activating the PI3K/AKT signaling pathway can regulate DcR3 expression and then promotes cell proliferation and migration and inhibit apoptosis. ${ }^{22}$ Down-regulation of IL33 reduced cell viability and EMT and induced cell apoptosis by activating the PI3K/AKT signaling pathway. ${ }^{23}$ It is not yet clear whether CD147, DcR3, and IL33 are involved in the process of KD. So we investigated their serum levels in different stages of $\mathrm{KD}$ compared to healthy controls. And we explored these pro-inflammatory factors' function in the pathophysiology and prognosis of $\mathrm{KD}$.

\section{Materials and Methods}

\section{Ethical Statement}

The ethical committee of the Children's Hospital, Zhejiang University School of Medicine, approved the study. The investigation, which was based on the institution's guidelines for human studies, conformed to the ethics guidelines of the Declaration of Helsinki. Informed consent was obtained from each patient's parent or guardian.

\section{Patients}

Seventy-one patients with Kawasaki disease (50 boys and 21 girls) were enrolled in this study at the Children's Hospital, Zhejiang University School of Medicine, China, between 2015 and 2017. All patients fulfilled the diagnostic criteria ${ }^{1}$ and were treated with high-dose intravenous immunoglobulin (IVIG) $(2 \mathrm{~g} / \mathrm{kg})$ and oral Aspirin at 30 to $50 \mathrm{mg} / \mathrm{kg} / \mathrm{d}$. When the patient's temperature returned to normal for three days, the dose of Aspirin was reduced to 3 to $5 \mathrm{mg} / \mathrm{kg} / \mathrm{d}$ for eight weeks. There were eight IVIGresistant $\mathrm{KD}$ patients whose temperature was still higher than $38^{\circ} \mathrm{C}$ after 36 hours of standard treatment, so they were continued on IVIG at $1 \sim 2 \mathrm{~g} / \mathrm{kg} / \mathrm{d}$ or methylprednisolone therapy. Nine patients, including seven girls and two boys, had CALs (defined as coronary artery z-score $>2.0)^{1}$ identified by echocardiography during the disease process. Four patients had CALs in the left and right coronary arteries, four in the left coronary artery, and only one in the right. The complications of CALs in the seven patients were improved observably and disappeared in 3 months after standard treatment. Two patients have persisted with CALs for more than 9 and 12 months. Besides, there were 66 healthy children enrolled in the control group. All the KD patients and healthy children had no particular family history or general history, and there was no recurrence case. 


\section{Laboratory Analysis}

We, respectively, collected blood samples from healthy children and KD patients at several time points. The first time point was at the acute phase of the disease before IVIG treatment (Pre-IVIG). The second was three days after the patients' temperature had returned to regular following IVIG treatment (Post-IVIG). And the third was at 14 to 21 days when the patients were first followed up after discharge (about one month of the disease). The samples were centrifuged to yield serum immediately and then kept at $-80^{\circ} \mathrm{C}$ until analysis.

We measured serum levels of CD147, DcR3, and IL33 according to the manufacturer's instructions with Enzymelinked Immunosorbent Assay Kit (ELISA). Our hospital laboratory used conventional methods to measure IL6 and IL10 and WBC, CRP, and ESR. We used the following reagents: human EMMPRIN/CD147 immunoassay (catalog number DEMP00, Quantikine, R\&D Systems, Minneapolis, MN, USA), human DcR3 ELISA Kit (catalog number ELHDcR3 RayBiotech, US); human IL33 immunoassay (catalog number D3300, Quantikine, R\&D Systems, Minneapolis, MN, USA).

\section{Statistical Analysis}

We used the SPSS22.0 software package for all statistical analyses and GraphPad Prism 9 for painting the figures. Except where indicated otherwise, the results were expressed as mean $(95 \% \mathrm{CI})$. Routine data analysis was performed with analysis of variance (ANOVA) followed by least significant difference (LSD) test for multiple comparisons; nonparametric data were analyzed by the Mann-Whitney test. Differences in parameters were considered Statistic significant at P-values less than 0.05 .

We used GeneMANIA and Cytoscape APP to see the interactions between protein and protein and predict gene function. The query gene list included CD147, DCR3, IL33, VEGF, VEGI, ZO-1, ICAM1, S100A12, TNFa, IL6, and IL10. At the same time, the protein list was CD147, DCR3, IL33, and expanded necessary.

\section{Results}

\section{Clinical Characteristics of KD Patients}

Our study enrolled $71 \mathrm{KD}$ patients (50 boys and 21 girls) and 66 healthy children ( 33 boys and 33 girls). There were no significant differences in age and weight between KD patients and healthy control. The duration of fever days was $2-15$ days in KD patients. Almost all KD patients had cervical lymphadenopathy, but 28 cases did not have extremity changes (Figure 1A). Twenty-nine KD children did not have other clinical findings until discharge. Ten patients had more than one other manifestation (Figure 1B). Eight cases were IVIG non-responders, while nine KD patients got CALs, but they did not overlap.

\section{Serum Levels of CDI47, DcR3, and IL33 with KD Patients and Healthy Controls}

We summarized the clinical characteristics of $71 \mathrm{KD}$ patients and 66 healthy children in Table 1 and Figure 2. Serum levels of CD147, DcR3, and IL33 were significantly increased, like the change of CRP, ESR, IL6, and IL10 in KD patients before IVIG treatment. Serum levels of CD147, DcR3, and IL33

A
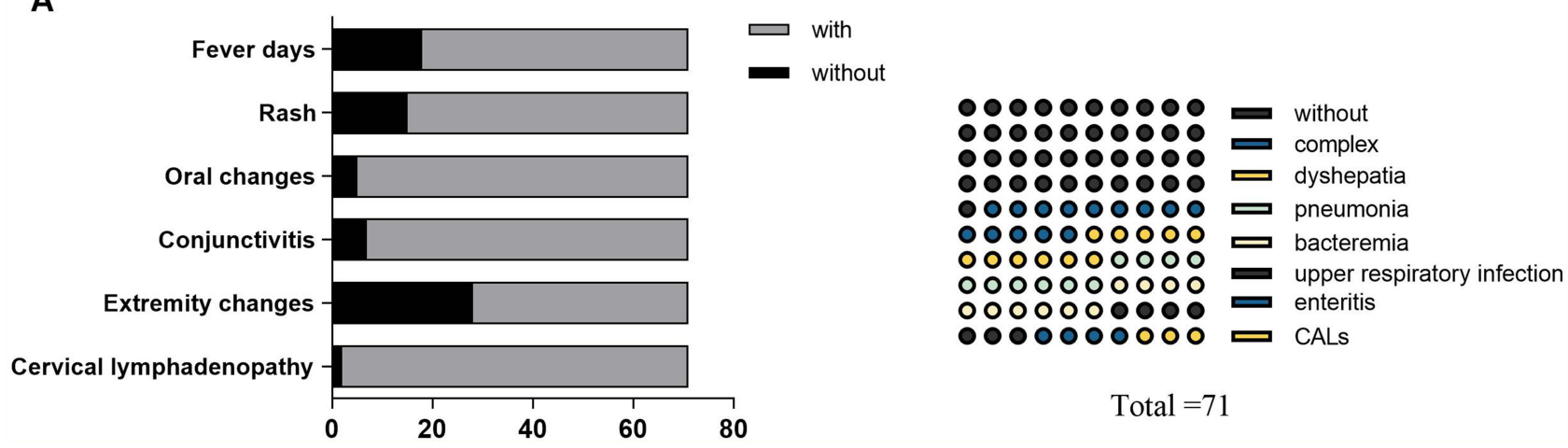

Figure I The clinical features of KD patients. (A) The main clinical characters. With fever days: fever days more than five. (B) Other clinical findings of KD patients. Complex: two or more than two clinical findings. 
Table I Serum Levels of CDI47, DcR3, and IL33 with KD Patients and Healthy Controls

\begin{tabular}{|c|c|c|c|c|}
\hline \multicolumn{5}{|c|}{ Patients with Kawasaki Disease } \\
\hline & Controls & Pre-IVIG & Post-IVIG & One Month \\
\hline Male/female & $33 / 33^{\$}$ & $50 / 21$ & & \\
\hline Age, Month & $34.1(28.0 \sim 40.2)$ & $38.6(32.4 \sim 44.7)$ & & \\
\hline Weight, $\mathrm{Kg}$ & $14.9(\mid 3.2 \sim 16.6)$ & $14.8(\mid 3.4 \sim 16.6)$ & & \\
\hline $\mathrm{CD} \mid 47, \mathrm{ng} / \mathrm{mL}$ & $6.4 \mathrm{I}(6.21 \sim 6.6 \mathrm{I})^{*}$ & $8.96(8.85 \sim 9.06)^{\#}$ & $8.47(8.34 \sim 8.60)^{\&}$ & $7.86(7.73 \sim 7.99)$ \\
\hline DcR3, ng/mL & $1.76(1.67 \sim 1.84)^{*}$ & $2.62(2.5 \mathrm{I} \sim 2.74)^{\#}$ & $2.30(2.20 \sim 2.40)^{8}$ & $2.05(1.96 \sim 2.14)$ \\
\hline $\mathrm{IL} 33,10^{2} \mathrm{pg} / \mathrm{mL}$ & $1.18(1.16 \sim 1.21) *$ & I.57 (I.53 1.60) $)^{\#}$ & $1.48(1.45 \sim 1.52)^{8}$ & $1.36(1.33 \sim 1.40)$ \\
\hline WBC, $10^{9} / \mathrm{L}$ & $8.96(8.38 \sim 9.54)^{* *}$ & $14.7(13.2 \sim 16.2)^{\#}$ & $8.07(7.21 \sim 8.92)$ & 7.53 (6.89 8.17) \\
\hline CRP, mg/L & $2.85(2.32 \sim 3.38)^{*}$ & $74.2(6 \mathrm{I} .7 \sim 86.8)^{\#}$ & $16.3(\mid 1.4 \sim 21.2)^{\&}$ & $2.72(1.82 \sim 3.63)$ \\
\hline $\mathrm{ESR}, \mathrm{mm} / \mathrm{h}$ & & $72.5(64.4 \sim 80.6)^{\#}$ & $94.8(87.4 \sim 102)^{\&}$ & $19.33(15.1 \sim 23.6)$ \\
\hline $\mathrm{IL} 6,10^{2} \mathrm{pg} / \mathrm{mL}$ & $0.12(0.07 \sim 0.17)^{*}$ & $0.99(0.62 \sim 1.36)$ & & \\
\hline ILI0, pg/mL & $4.89(3.36 \sim 6.42)^{*}$ & $17.9(12.3 \sim 23.5)$ & & \\
\hline
\end{tabular}

Note: Values are the mean $(95 \% \mathrm{Cl}) .{ }^{\$} \mathrm{P}$-values $<0.05$ between $\mathrm{KD}$ patients and healthy control. *All P-values are for patients versus healthy control, and all of them $<$ 0.01 . **P-values $<0.0 \mathrm{I}$ is for group Pre-IVIG versus healthy control. ${ }^{\#} \mathrm{P}$-values $<0.0 \mathrm{I}$ in kinds of groups with Kawasaki disease. ${ }^{\&} \mathrm{P}$-values $<0.0 \mathrm{I}$ in kinds of groups. Abbreviations: IVIG, intravenous immunoglobulin; CD 147, cluster of differentiation I47; DcR3, decoy receptor 3; IL33, interleukin 33; WBC, white blood cell; CRP, C-reactive protein; ESR, erythrocyte sedimentation rate; IL6, interleukin 6; ILI0, interleukin I0.

gradually decreased over time after the treatment of IVIG. However, in different stages with KD patients, even at one month, serum levels of CD147, DcR3, and
IL33 were significantly higher than healthy controls. Primarily, only fewer cases of CD 147, DcR3, and IL33 levels increased after IVIG treatment.

\section{A}
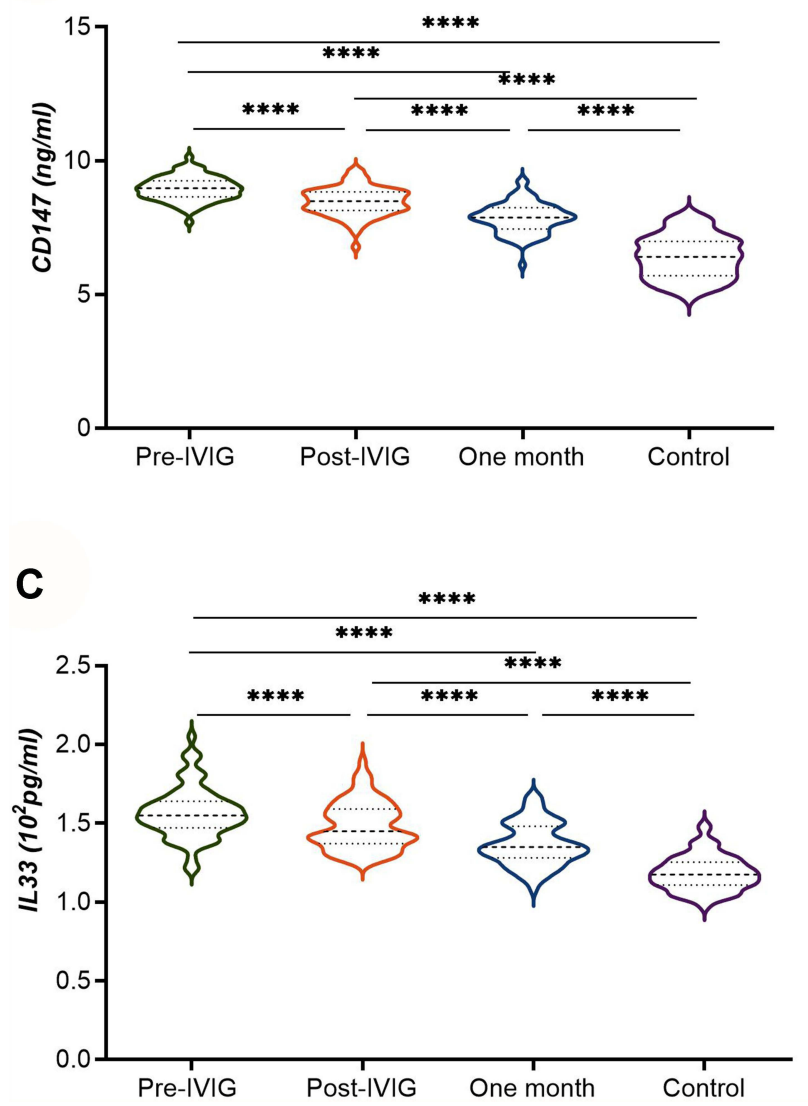

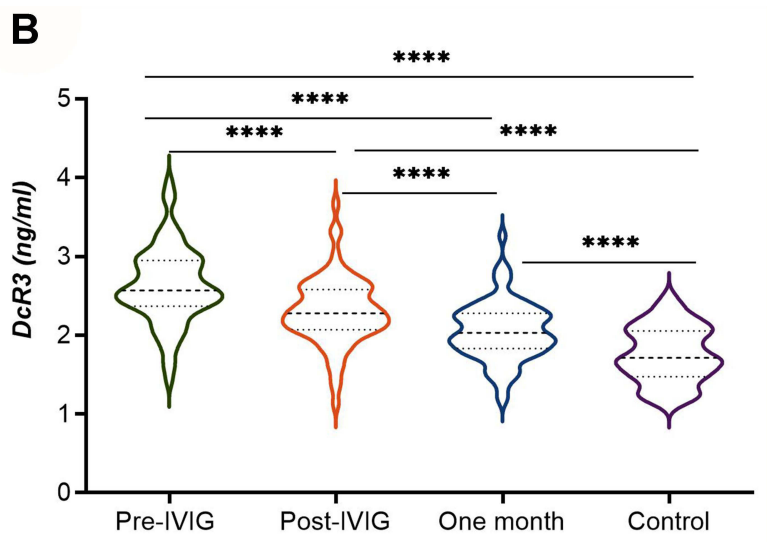

D

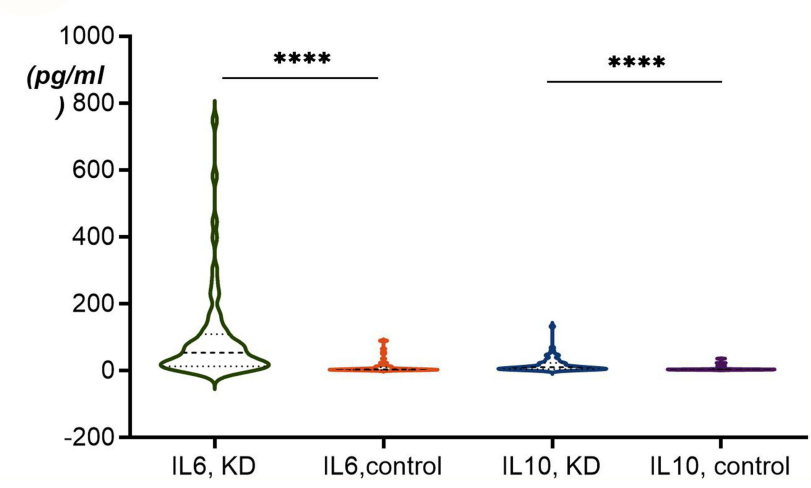

Figure 2 Serum levels of CDI47, DcR3, IL33, IL6, ILI0 with KD patients and healthy controls. (A-D) $* * * *$ P-values $<0.000$ I in kinds of groups. 
Serum Levels of CDI47, DcR3, and IL33 in IVIG Responders and Non-Responders with KD Patients (Table 2 and Figure 3A) Before treatment with IVIG, DcR3 and CD147 were slightly higher in IVIG responders than IVIG nonresponders. In contrast, the levels of IL33, IL6, and IL10 were marginally lower in IVIG responders versus IVIG non-responders. There were no statistical differences between group IVIG responders and group IVIG nonresponders. In each group, CD147, DcR3, and IL33 decreased according to the rule of time. The conventional indexes had similar conditions to them. Before IVIG treatment, CRP in group IVIG non-responders was higher than group responders, and $\mathrm{P}$-value $<0.05$. However, after IVIG, ESR increased and P-value $<0.05$ compared to group Pre-IVIG. Though the mean age and weight in group IVIG non-responders were higher than the other group, there was no statistical difference.

Serum Levels of CDI47, DcR3, and IL33 in KD Patients without CALs and with CALs (Table 3 and Figure 3B)

Nine patients were complicated CALs, and 62 cases were without CALs. And the total incidence of CALs was

Table 2 Serum Levels of CDI47, DcR3, and IL33 in IVIG Responders and Non-Responders with KD Patient

\begin{tabular}{|c|c|c|c|}
\hline & & IVIG Responders & IVIG Non-Responders \\
\hline & & $(n=63)$ & $(n=8)$ \\
\hline \multicolumn{2}{|l|}{ Male/female } & $44 / 19$ & $6 / 2$ \\
\hline \multicolumn{2}{|l|}{ Age, Month } & $32.2(25.6 \sim 38.8)$ & $49.1(34.3 \sim 64.0)$ \\
\hline \multicolumn{2}{|l|}{ Weight, $\mathrm{Kg}$} & $14.3(12.5 \sim 16.1)$ & $19.5(12.9 \sim 26.1)$ \\
\hline \multirow[t]{3}{*}{$\mathrm{CD} \mid 47, \mathrm{ng} / \mathrm{mL}$} & Pre-IVIG & $8.98(8.87 \sim 9.10)$ & $8.74(8.42 \sim 9.07)$ \\
\hline & Post-IVIG & $8.50(8.36 \sim 8.63)$ & $8.24(7.87 \sim 8.62)$ \\
\hline & One month & $7.85(7.7 \mid \sim 8.00)$ & 7.91 (7.53 8.29) \\
\hline \multirow[t]{3}{*}{$\mathrm{DcR3}, \mathrm{ng} / \mathrm{mL}$} & Pre-IVIG & $2.65(2.53 \sim 2.78)$ & $2.40(2.11 \sim 2.68)$ \\
\hline & Post-IVIG & $2.33(2.22 \sim 2.44)$ & $2.09(2.87 \sim 2.3 \mathrm{I})$ \\
\hline & One month & 2.09 (1.98 2.17) & $1.81(1.61 \sim 2.02)$ \\
\hline \multirow[t]{3}{*}{ IL33, $10^{2} \mathrm{pg} / \mathrm{mL}$} & Pre-IVIG & $1.56(1.52 \sim 1.60)$ & $1.62(1.47 \sim 1.77)$ \\
\hline & Post-IVIG & $1.48(\mathrm{I} .44 \sim 1.5 \mathrm{I})$ & $1.55(1.39 \sim 1.70)$ \\
\hline & One month & $1.36(1.32 \sim 1.39)$ & $1.41(1.30 \sim 1.53)$ \\
\hline \multirow[t]{3}{*}{ WBC, $10^{9} / \mathrm{L}$} & Pre-IVIG & $14.8(13.2 \sim 16.4)$ & $13.8(9.20 \sim 18.4)$ \\
\hline & Post-IVIG & $7.98(7.09 \sim 8.86)$ & $8.73(4.62 \sim 12.8)$ \\
\hline & One month & $7.56(6.85 \sim 8.26)$ & $7.35(5.40 \sim 9.29)$ \\
\hline \multirow[t]{3}{*}{ CRP, mg/L } & Pre-IVIG & $69.8(56.9 \sim 82.7)$ & $109(62.2 \sim 155)^{*}$ \\
\hline & Post-IVIG & $14.5(\mid 0.2 \sim 18.8)$ & $29.7(-3.38 \sim 62.7)$ \\
\hline & One month & $2.45(1.55 \sim 3.42)$ & $4.33(0.54 \sim 8.13)$ \\
\hline \multirow[t]{3}{*}{$\mathrm{ESR}, \mathrm{mm} / \mathrm{h}$} & Pre-IVIG & $92.2(85.3 \sim 99.1)$ & II 4 (70.8 157) \\
\hline & Post-IVIG & $17.3(\mid 4.2 \sim 20.4)$ & $34.2(1.78 \sim 66.7)^{*}$ \\
\hline & One month & $14.8(\mid 3.2 \sim 16.4)$ & |3.8 (9.20 |8.4) \\
\hline $\mathrm{IL} 6,10^{2} \mathrm{pg} / \mathrm{mL}$ & Pre-IVIG & $0.98(0.58 \sim 1.37)$ & I.II (0.4I 2.64) \\
\hline ILI0, pg/mL & Pre-IVIG & I5.4 (II.I 19.6) & $39.8(0.10 \sim 89.9)$ \\
\hline
\end{tabular}

Note: Values are the mean $(95 \% \mathrm{Cl})$. *P-value $<0.05$. 
A

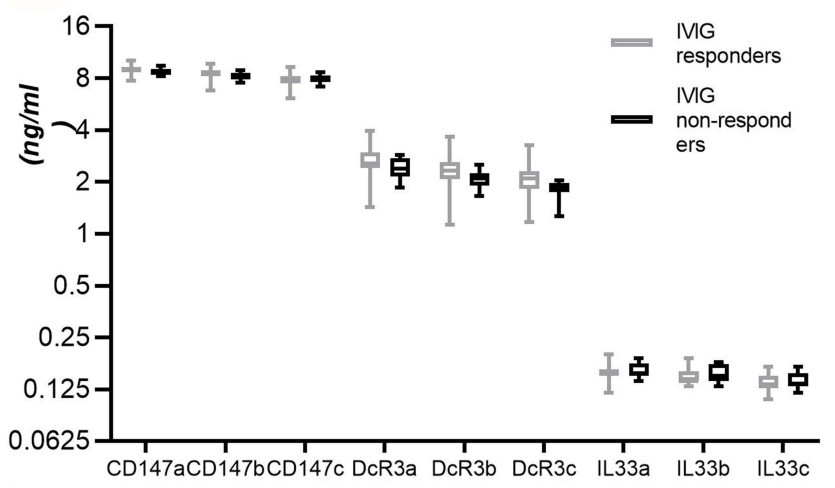

B

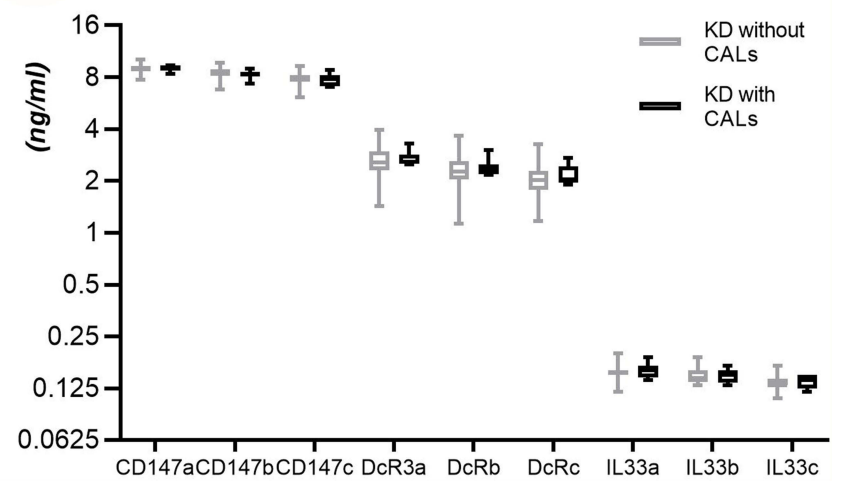

Figure 3 Serum levels of CDI47, DcR3, and IL33 with KD patients. (A) Compared in groups IVIG responders and non-responders. (B) Compared in groups without CALs and with CALs.

Notes: a. Pre-IVIG, b. Post-IVIG, c. One month. P-values $>0.05$ between groups. P-values $>0.05$ between groups IVIG responders and groups IVIG non-responders.

$12.7 \%$. Besides, the age of KD patients with CALs less than patients without CALs, and the P-value was $<0.05$. Before treatment with IVIG, CD147 and DcR3 was slightly higher in group KD with CALs than those without CALs. In contrast, the levels of IL33, IL6, and IL10 were marginally lower in groups with CALs versus without CALs. There were no statistical differences between groups without CALs and with CALs. In each group, DcR3, IL33, CD147 decreased the levels according to the rule of time.

\section{The Interactions Between Protein and Protein and Prediction of Gene Function (Figures 4 and 5)}

A query gene list including CD147 (BSG), DCR3 (TNFRSF6B), IL33, VEGF, VEGI, ZO-1, ICAM1, S100A12, TNFa, IL6, and IL10, GeneMANIA found functionally similar genes using a wealth of genomics and proteomics data. We used GeneMANIA and Cytoscape APP to see the interactions between protein and protein and predict gene function. The networks represented coexpression 50.84\%, Physical interactions 27.4\%, predicted $15.33 \%$, co-localization $3.57 \%$, shared protein domains $2.82 \%$, and genetic interations $0.3 \%$. IL33 had more tasks than shown in Figure 4. There was no direct correlation with protein CD147, DcR3, and IL33, and they made a protein interaction network through other related proteins (Figure 5).

\section{Discussion}

As a clinical syndrome, Kawasaki disease (KD) has various atypical clinical manifestations. Although the scientific statement of AHA in $2017^{1}$ provided us with a diagnosis process of incomplete $\mathrm{KD}$, the clinical variability has still increased the difficulty in the diagnosis of KD. Our result demonstrated that almost all patients were complicated with cervical lymphadenopathy, but one-third were without extremity changes. Only $29 \mathrm{KD}$ children did not have other clinical findings until discharge; moreover, ten patients had more than one other manifestation. The coronary artery damage may even be challenging to recover. And the high incidence of CALs may be the result of regional bias. However, we found KD CALs increased in clinical practice recently. Besides, age is a risk factor for CALs.

Furthermore, the mechanism of KD remains unclear, and people have paid more and more attention to $\mathrm{KD}$ because of its severe complications. Although there are many proinflammatory factors to be conformed related to $\mathrm{KD}$, no study told us the definite factor which can determine the development of KD. We selected several cell markers associated with VEGF or endothelial cell or heart injury indicators from the literature, measured the difference in serum expression levels between KD children and normal controls. Our study showed that the serum levels of CD147, DcR3, and IL33 with KD patients peaked in the acute stage before IVIG treatment and decreased gradually after IVIG and in the subacute phase one month later. The application steadily reduced, but the three periods were higher than the healthy control, and there were statistical differences. Studies showed plasma IL33 significantly increased after IVIG treatment with immunodeficiency or autoimmune disease. ${ }^{24,25}$ It is different from our research because the disorders and the conditions of patients were unconformities, and their number 
Table 3 Serum Levels of CDI47, DcR3, and IL33 in KD Patients without CALs and with CALs

\begin{tabular}{|c|c|c|c|}
\hline & & KD without CALs & KD with CALs \\
\hline & & $(n=62)$ & $(n=9)$ \\
\hline \multicolumn{2}{|l|}{ Male/female } & $42 / 20$ & $7 / 2$ \\
\hline \multicolumn{2}{|l|}{ Age, Month } & $36.8(30.1 \sim 43.4)$ & $16.0(5.39 \sim 26.6)^{*}$ \\
\hline \multicolumn{2}{|l|}{ Weight, $\mathrm{Kg}$} & $15.4(13.5 \sim 17.3)$ & II.3 (8.37 |4.3) \\
\hline \multirow[t]{3}{*}{ DcR3, ng/mL } & Pre-IVIG & 2.61 (2.48 2.74) & 2.71 (2.50 2.92) \\
\hline & Post-IVIG & $2.29(2.17 \sim 2.40)$ & $2.39(2.18 \sim 2.60)$ \\
\hline & One month & $2.03(1.93 \sim 2.13)$ & $2.17(\mathrm{I} .92 \sim 2.4 \mathrm{I})$ \\
\hline \multirow[t]{3}{*}{ IL33, $10^{2} \mathrm{pg} / \mathrm{mL}$} & Pre-IVIG & $1.57(1.53 \sim 1.61)$ & $1.59(1.45 \sim 1.72)$ \\
\hline & Post-IVIG & $1.48(1.45 \sim 1.52)$ & $1.49(1.37 \sim 1.60)$ \\
\hline & One month & $1.36(1.33 \sim 1.39)$ & $1.39(1.28 \sim 1.50)$ \\
\hline \multirow[t]{3}{*}{ CDI47, ng/mL } & Pre-IVIG & $8.95(8.83 \sim 9.07)$ & $9.00(8.78 \sim 9.23)$ \\
\hline & Post-IVIG & $8.49(8.36 \sim 8.63)$ & 8.31 (7.95 8.67) \\
\hline & One month & $7.87(7.73 \sim 8.01)$ & $7.78(7.28 \sim 8.28)$ \\
\hline \multirow[t]{3}{*}{ WBC, $10^{9} / \mathrm{L}$} & Pre-IVIG & $14.4(\mid 2.8 \sim 15.9)$ & $17.2(\mid 1.7 \sim 22.7)$ \\
\hline & Post-IVIG & $7.99(7.04 \sim 8.95)$ & $8.60(6.53 \sim 10.7)$ \\
\hline & One month & $7.66(6.96 \sim 8.36)$ & $6.64(4.88 \sim 8.39)$ \\
\hline \multirow[t]{3}{*}{ CRP, mg/L } & Pre-IVIG & $77.2(63.3 \sim 100)$ & $54.6(24.7 \sim 84.4)$ \\
\hline & Post-IVIG & $16.7(1 \mid .2 \sim 22.2)$ & I3.5 (3.96 23.0) \\
\hline & One month & $2.93(1.91 \sim 3.95)$ & $1.33(0.48 \sim 2.19)$ \\
\hline \multirow[t]{3}{*}{$\mathrm{ESR}, \mathrm{mm} / \mathrm{h}$} & Pre-IVIG & 73.1 (64.4 8I.8) & $68.6(40.6 \sim 96.5)$ \\
\hline & Post-IVIG & $94.8(86.5 \sim 103)$ & $95.0(77.2 \sim 113)$ \\
\hline & One month & $20.2(15.5 \sim 25.0)$ & $13.0(5.43 \sim 20.6)$ \\
\hline IL6, $10^{2} \mathrm{pg} / \mathrm{mL}$ & Pre-IVIG & I.II (0.67 I.53) & $0.35(0.13 \sim 0.58)$ \\
\hline ILI0, pg/mL & Pre-IVIG & $19.6(13.1 \sim 26.1)$ & $8.30(2.92 \sim 13.7)$ \\
\hline
\end{tabular}

Note: Values are the mean $(95 \% \mathrm{Cl})$. $* \mathrm{P}$-value $<0.05$.

of cases was insufficient. There were no statistical differences between group IVIG responders and IVIG nonresponders or between groups without CALs and with CALs.

GeneMANIA (http://genemania.org) is a flexible, friendly website for generating hypotheses about gene tasks, analyzing and prioritizing genes for functional assays. ${ }^{26}$ We explore the functions of CD147, DcR3, and IL33 from GeneMANIA, such as leukocyte migration, myeloid leukocyte migration, regulation of chemokine production, cell chemotaxis, chemokine production, cellular response to tumor necrosis factor, restriction of the interleukin-6 output, and so on. These third pro- inflammatory factors were coexpressed, physical interactions, genetic interactions with other KD-related factors. There was no direct correlation with protein CD147, DcR3, and IL33, and they made a protein interaction network through other related proteins.

Since some studies discovered CD147 to play a pivotal role in the complex processes of atherogenesis and acute plaque rupture and thrombosis, ${ }^{27}$ but our result revealed no statistical differences between groups without CALs and with CALs for serum CD 147. We have described that levels of IL6, IL10, TNF $\alpha$, and IFN $\gamma$ were significantly increased in KD patients Pre-IVIG, while Post-IVIG, the levels of IL6, 


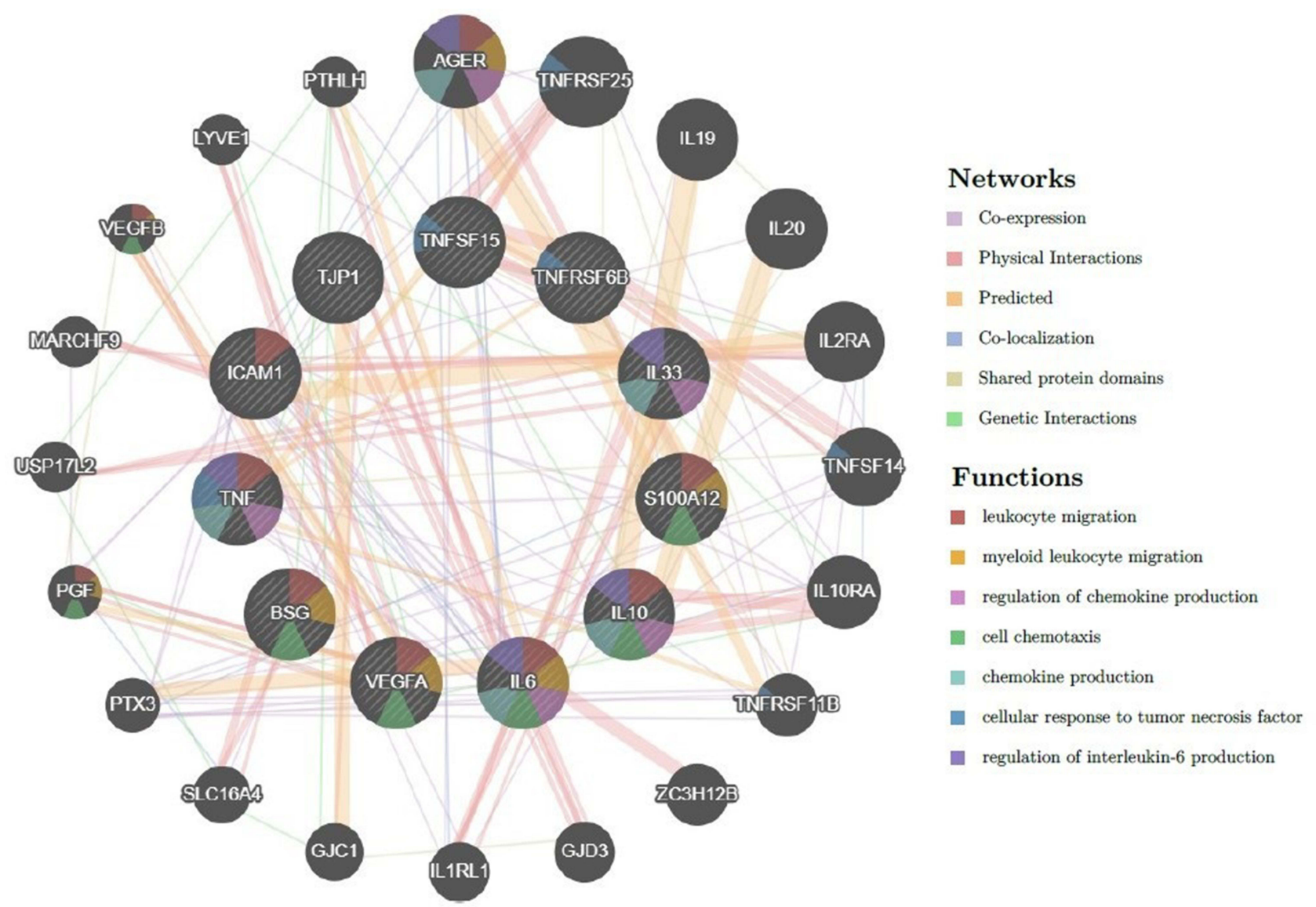

Figure 4 The interactions between gene and gene and prediction of gene function.

Abbreviations: BSG, basigin (CD I47); TNFRSF6B (DcR3), TNF receptor superfamily member 6b; IL33, interleukin 33; TNFSFI5, TNF superfamily member I5; SI00AI2, SI00 calcium binding protein AI2; ILI0, interleukin I0; IL6, interleukin 6; VEGFA, vascular endothelial growth factor A; TNF, tumor necrosis factor; ICAMI, intercellular adhesion molecule I; TJPI, tight junction protein I; AGER, advanced glycosylation end-product specific receptor; TNFRSF25, TNF receptor superfamily member 25; ILI9, interleukin 19; IL20, interleukin 20; IL2RA, interleukin 2 receptor subunit alpha; TNFSFI4, TNF superfamily member I4; ILIORA, interleukin I0 receptor subunit alpha; TNFRSFI IB, TNF receptor superfamily member I Ib; ZC3HI2B, zinc finger CCCH-type containing I2B; GJD3, gap junction protein delta 3; ILIRLI, interleukin I receptor like I; GJCI, gap junction protein gamma I; SLCI6A4, solute carrier family 16 member 4; PTX3, pentraxin 3; PGF, placental growth factor; USPI7L2, ubiquitin-specific peptidase 17 like family member 2; MARCHF9, membrane associated ring-CH-type finger 9; VEGFB, vascular endothelial growth factor B; LYVEI, lymphatic vessel endothelial hyaluronan receptor I; PTHLH, parathyroid hormone like hormone.

IL10, and IFN $\gamma$ quickly decreased. ${ }^{28}$ IL33 had the same rule as other interleukins, but it does not predict IVIG nonresponders or CALs.

Infliximab (IFX) is a novel chimeric monoclonal antibody and produces anti-inflammatory effects through specific blocking of tumor necrosis factor-alpha (TNF- $\alpha$ ). ${ }^{29}$ IFX is a vital pro-inflammatory factor significantly elevated in the blood circulation of children with $\mathrm{KD} .{ }^{30}$ The effectiveness of IFX in combination with IVIG treatment of nonreactive type KD is controversial. A retrospective review of 2 centers suggested that patients receiving infliximab had shorter hospitalization and fewer days of fever. Still, coronary artery outcomes and adverse events were similar. ${ }^{31}$ We also found the same event in the clinical practice. Perhaps because infliximab is only an antagonist of TNFa and only works on the inflammatory response pathway. IVIG and glucocorticosteroids are multi-acting mechanisms for vasculitis. But a metaanalysis in 2021 emphasizes the importance of IFX on the treatment response in high-risk KD patients. IFX may play a role in Asian KD patients, prevent progressive $\mathrm{CAA}$, and not increase the risk of adverse events in $\mathrm{KD}$ patients. $^{32}$

There is no definite signaling pathway in KEGG PATHWAY Database about Kawasaki disease. But all of CD147, DcR3, and IL33 are associated with PI3K/AKT signaling pathways. A study said that the PTEN/PI3K/VEGF signaling course plays a role in developing KD. ${ }^{19}$ PI3K-Akt signaling pathway can regulate fundamental cellular functions like transcription, translation, proliferation, growth, and survival. $^{20}$ So, we should study deeply between KD and these pro-inflammatory factors. 


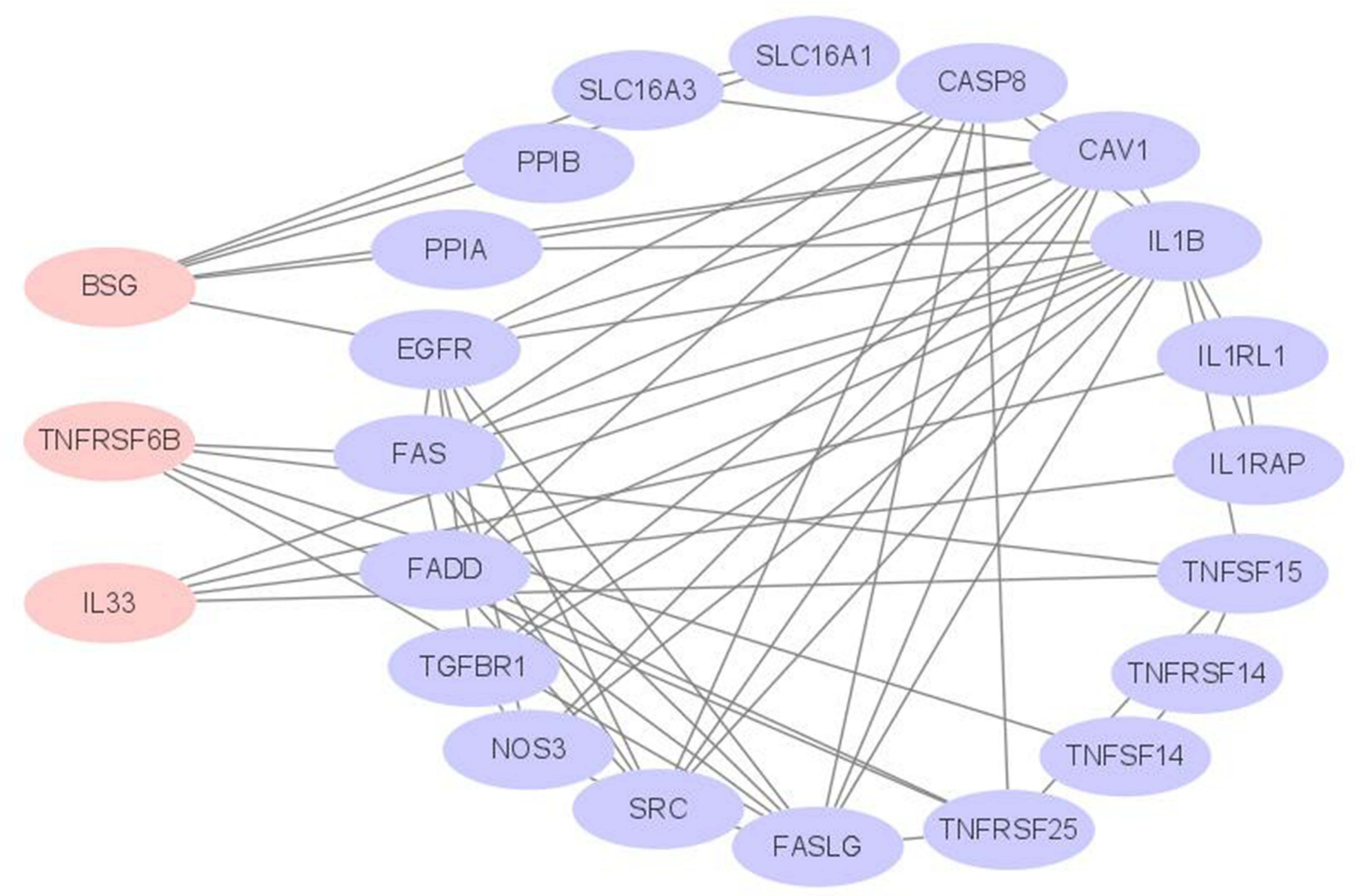

Figure 5 The interactions between protein and protein with CDI47, DcR3, and IL33.

Abbreviations: BSG, basigin (CD 147); TNFRSF6B (DcR3), TNF receptor superfamily member 6b; IL33, interleukin 33; CASP8, Caspase-8; PPIA, Peptidyl-prolyl cis-trans isomerase A; PPIB, Peptidyl-prolyl cis-trans isomerase B; FAS, Tumor necrosis factor receptor superfamily member 6; FADD, FAS-associated death domain protein; ILIRLI, Interleukin-I receptor-like I; ILIRAP, Interleukin I receptor accessory protein; ILIB, Interleukin-I beta; EGFR, Epidermal growth factor receptor; SRC, Proto-oncogene tyrosine-protein kinase Src; NOS3, Nitric oxide synthase, endothelial; TGFBRI, TGF-beta receptor type-I; CAVI, Caveolin-I; SLCI6AI, Monocarboxylate transporter I; SLCI6A3, Monocarboxylate transporter 4; TNFSFI4, Tumor necrosis factor ligand superfamily member I4; TNFRSFI4, Tumor necrosis factor receptor superfamily member 14; TNFSFI5, Tumor necrosis factor ligand superfamily member 15; TNFRSF25, Tumor necrosis factor receptor superfamily member 25; FASLG, Tumor necrosis factor ligand superfamily member 6 .

We put forward a hypothesis: KD inflammatory storms and vascular damage are two pathways, not entirely coincide, and we did not find the coefficient intersection yet. Inflammation is just an incentive to activate the target; once vascular damage starts, how to terminate the process still needs further study. Applying bioinformatics technology to the next step of research and exploring the pathogenesis of Kawasaki disease from multiple omics in vitro and animal models is our next research direction.

\section{Conclusion}

In conclusion, KD patients' serum CD147, DcR3, and IL33 peaked in the acute stage before IVIG treatment and decreased gradually after IVIG and in the subacute phase one month later. It suggests that the inflammatory pathway is involved in the pathophysiology of $\mathrm{KD}$, which provides novel evidence for diagnosing and treating $\mathrm{KD}$ with its inflammatory inhibitors.

\section{Acknowledgments}

This work is supported, in part, by grants from The National Natural Science Foundation of China (No. 81670251, 81970434), Medicine and Health Science and Technology Project of Zhejiang Province (No. 2018260214).

\section{Disclosure}

The authors report no conflicts of interest in this work.

\section{References}

1. McCrindle BW, Rowley AH, Newburger JW, et al. Diagnosis, treatment, and long-term management of Kawasaki disease: a scientific statement for health professionals from the American Heart Association. Circulation. 2017;135(17):e927-e999. doi:10.1161/ CIR.0000000000000484

2. Matsubara T, Ichiyama T, Furukawa S. Immunological profile of peripheral blood lymphocytes and monocytes/macrophages in Kawasaki disease. Clin Exp Immunol. 2005;141(3):381-387. doi:10.1111/j.1365-2249.2005.02821.x 
3. Franco A, Shimizu C, Tremoulet AH, Burns JC. Memory T-cells and characterization of peripheral T-cell clones in acute Kawasaki disease. Autoimmunity. 2010;43(4):317-324. doi:10.3109/08916930903405891

4. Muramatsu T. Basigin (CD147), a multifunctional transmembrane glycoprotein with various binding partners. J Biochem. 2016;159 (5):481-490. doi:10.1093/jb/mvv127

5. Li K, Nowak RA. The role of basigin in reproduction. Reproduction. 2020;159:R97-R109.

6. Zong J, Li Y, Du D, Liu Y, Yin Y. CD147 induces up-regulation of vascular endothelial growth factor in U937-derived foam cells through PI3K/AKT pathway. Arch Biochem Biophys. 2016;60 9:31-38. doi:10.1016/j.abb.2016.09.001

7. Kurosawa R, Satoh K, Nakata T, et al. Identification of celastrol as a novel therapeutic agent for pulmonary arterial hypertension and right ventricular failure through suppression of Bsg (Basigin)/CyPA (Cyclophilin A). Arterioscler Thromb Vasc Biol. 2021;41(3):12 05-1217. doi:10.1161/ATVBAHA.120.315731

8. Rahat MA, Safieh M, Simanovich E, et al. The role of EMMPRIN/ CD147 in regulating angiogenesis in patients with psoriatic arthritis. Arthritis Res Ther. 2020;22(1):240. doi:10.1186/s13075-020-02333-6

9. Weng SC, Tarng DC. Role of prognostic biomarker decoy receptor 3 and immunomodulation in kidney diseases. J Chin Med Assoc. 2019;82(9):680-684. doi:10.1097/JCMA.0000000000000149

10. Migone TS, Zhang J, Luo X, et al. TL1A is a TNF-like ligand for DR3 and TR6/DcR3 and functions as a T cell costimulator. Immunity. 2002;16(3):479-492. doi:10.1016/S1074-7613(02)00283-2

11. Li XY, Hou HT, Chen HX, Wang ZQ, He GW. Increased circulating levels of tumor necrosis factor-like cytokine 1A and decoy receptor 3 correlate with SYNTAX score in patients undergoing coronary surgery. J Int Med Res. 2018;46(12):5167-5175. doi:10.1177/0300060518793787

12. Chen X, Wang R, Chen W, Lai L, Li Z. Decoy receptor-3 regulates inflammation and apoptosis via PI3K/AKT signaling pathway in coronary heart disease. Exp Ther Med. 2019;17(4):2614-2622.

13. Lohning M, Stroehmann A, Coyle AJ, et al. T1/ST2 is preferentially expressed on murine Th2 cells, independent of interleukin 4, interleukin 5, and interleukin 10, and important for Th2 effector function. Proc Natl Acad Sci U S A. 1998;95(12):6930-6935. doi:10.1073/pnas.95.12.6930

14. Schmitz J, Owyang A, Oldham E, et al. IL33, an interleukin-1-like cytokine that signals via the IL-1 receptor-related protein ST2 and induces $\mathrm{T}$ helper type 2-associated cytokines. Immunity. 2005;23 (5):479-490. doi:10.1016/j.immuni.2005.09.015

15. Allakhverdi Z, Smith DE, Comeau MR, Delespesse G. Cutting edge: the ST2 ligand IL33 potently activates and drives maturation of human mast cells. J Immunol. 2007;179(4):2051-2054. doi:10.4049/ jimmunol.179.4.2051

16. Iikura M, Suto H, Kajiwara N, et al. IL33 can promote survival, adhesion and cytokine production in human mast cells. Lab Invest. 2007;87(10):971-978. doi:10.1038/labinvest.3700663

17. Stojkovic S, Kaun C, Heinz M, et al. Interleukin-33 induces urokinase in human endothelial cells - possible impact on angiogenesis. J Thromb Haemost. 2014;12(6):948-957. doi:10.1111/jth.12581

18. Drube S, Kraft F, Dudeck J, et al. MK2/3 are pivotal for IL33-induced and mast cell-dependent leukocyte recruitment and the resulting skin inflammation. $J$ Immunol. 2016;197(9):36 62-3668. doi:10.4049/jimmunol.1600658
19. An X, Lv H, Tian J, He X, Ling N. Role of the PTEN/PI3K/VEGF pathway in the development of Kawasaki disease. Exp Ther Med. 2016;11(4):1318-1322. doi:10.3892/etm.2016.3026

20. Singh SS, Yap WN, Arfuso F, et al. Targeting the PI3K/Akt signaling pathway in gastric carcinoma: a reality for personalized medicine? World J Gastroenterol. 2015;21(43):12261-12273. doi:10.3748/wjg. v21.i43.12261

21. Fang F, Wang L, Zhang S, et al. CD147 modulates autophagy through the PI3K/Akt/mTOR pathway in human prostate cancer PC-3 cells. Oncol Lett. 2015;9(3):1439-1443. doi:10.3892/ol.2015.2849

22. Zhong M, Qiu X, Liu Y, et al. TIPE regulates DcR3 expression and function by activating the PI3K/AKT signaling pathway in CRC. Front Oncol. 2020;10:623048. doi:10.3389/fonc.2020.623048

23. Wang S, Zhao G, Zhao S, Qiao Y, Yang H. The effects of interleukin-33 (IL33) on osteosarcoma cell viability, apoptosis, and epithelial-mesenchymal transition are mediated through the PI3K/ AKT pathway. Med Sci Monit. 2020;26:e920766.

24. Maddur MS, Stephen-Victor E, Das M, et al. Regulatory T cell frequency, but not plasma IL-33 levels, represents potential immunological biomarker to predict clinical response to intravenous immunoglobulin therapy. J Neuroinflammation. 2017;14(1):58. doi:10. 1186/s12974-017-0818-5

25. Tjon AS, van Gent R, Jaadar $H$, et al. Intravenous immunoglobulin treatment in humans suppresses dendritic cell function via stimulation of IL-4 and IL-13 production. J Immunol. 2014;192(12):5625-5634. doi:10.4049/jimmunol.1301260

26. Franz M, Rodriguez H, Lopes C, et al. GeneMANIA update 2018. Nucleic Acids Res. 2018;46(W1):W60-W64. doi:10.1093/nar/gky311

27. Pennings GJ, Kritharides L. CD147 in cardiovascular disease and thrombosis. Semin Thromb Hemost. 2014;40(7):747-755. doi:10. 1055/s-0034-1390001

28. Wang Y, Wang W, Gong F, et al. Evaluation of intravenous immunoglobulin resistance and coronary artery lesions in relation to Th1/ Th2 cytokine profiles in patients with Kawasaki disease. Arthritis Rheum. 2013;65(3):805-814. doi:10.1002/art.37815

29. Subedi S, Gong Y, Chen Y, Shi Y. Infliximab and biosimilar infliximab in psoriasis: efficacy, loss of efficacy, and adverse events. Drug Des Devel Ther. 2019;13:2491-2502. doi:10.2147/DDDT.S200147

30. Lu Z, Wang F, Lv H. Efficacy of infliximab in the treatment of Kawasaki disease: a systematic review and meta-analysis. Exp Ther Med. 2021;21(1):15.

31. Son MB, Gauvreau K, Burns JC, et al. Infliximab for intravenous immunoglobulin resistance in Kawasaki disease: a retrospective study. J Pediatr. 2011;158(4):644-649. doi:10.1016/j.jpeds.2010. 10.012

32. Li D, Li X, Dou W, Zheng Y. The effectiveness of infliximab for Kawasaki disease in children: systematic review and meta-analysis. Transl Pediatr. 2021;10(5):1294-1306. doi:10.21037/tp-20-482 


\section{Publish your work in this journal}

The Journal of Inflammation Research is an international, peerreviewed open-access journal that welcomes laboratory and clinical findings on the molecular basis, cell biology and pharmacology of inflammation including original research, reviews, symposium reports, hypothesis formation and commentaries on: acute/chronic inflammation; mediators of inflammation; cellular processes; molecular mechanisms; pharmacology and novel anti-inflammatory drugs; clinical conditions involving inflammation. The manuscript management system is completely online and includes a very quick and fair peerreview system. Visit http://www.dovepress.com/testimonials.php to read real quotes from published authors. 